\title{
The Spatial Extent of Epiretinal Electrical Stimulation in the Healthy Mouse Retina
}

\author{
Zohreh Hosseinzadeha,b Archana Jalligampala, ${ }^{a, b, c}$ Eberhart Zrenner ${ }^{a, b, d}$ \\ Daniel Lleweylln Rathbuna, ${ }^{a, b}$, \\ Institute for Ophthalmic Research, Centre for Ophthalmology, Eberhard Karls University, Tuebingen, \\ bWerner Reichardt Centre for Integrative Neuroscience (CIN), Tuebingen, 'Graduate Training Centre \\ of Neuroscience / International Max Planck Research School, Tuebingen, 'Bernstein Centre for \\ Computational Neuroscience Tuebingen, Tuebingen, Germany
}

\section{Key Words}

Electrical response function - Hysteresis - Retinal implant - Bionic vision - Electrical point spread function

\begin{abstract}
Background/Aims: Retinal prostheses use electrical stimulation to restore functional vision to patients blinded by retinitis pigmentosa. A key detail is the spatial pattern of ganglion cells activated by stimulation. Therefore, we characterized the spatial extent of network-mediated electrical activation of retinal ganglion cells (RGCs) in the epiretinal monopolar electrode configuration. Methods: Healthy mouse RGC activities were recorded with a micro-electrode array (MEA). The stimuli consisted of monophasic rectangular cathodic voltage pulses and cycling full-field light flashes. Results: Voltage tuning curves exhibited significant hysteresis, reflecting adaptation to electrical stimulation on the time scale of seconds. Responses decreased from 0 to $300 \mu \mathrm{m}$, and were also dependent on the strength of stimulation. Applying the Rayleigh criterion to the half-width at half-maximum of the electrical point spread function suggests a visual acuity limit of no better than 20/946. Threshold voltage showed only a modest increase across these distances. Conclusion: The existence of significant hysteresis requires that future investigations of electrical retinal stimulation control for such long-memory adaptation. The spread of electrical activation beyond $200 \mu \mathrm{m}$ suggests that neighbouring electrodes in epiretinal implants based on indirect stimulation of RGCs may be indiscriminable at interelectrode spacings as large as $400 \mu \mathrm{m}$.

\section{Introduction}

There are several visual prostheses that stimulate different parts of the visual pathway, such as the optic nerve [1] and the visual cortex [2-4]. Retinal stimulation, however, appears to possess more advantages than stimulation of other parts of the visual system [5]. Nevertheless, retinal implants remain far from a perfect solution. The in vitro study of

Zohreh Hosseinazdeh

Daniel Lleweylln Rathbun
Institute for Ophthalmic Research, Centre for Ophthalmology, Eberhard Karls University,

Tuebingen, Elfriede Aulhorn Str.7, 72076 Tübingen (Germany)

E-Mail zohreh.hosseinazdeh@uni-tuebingen.de/daniel.rathbun@uni-tuebingen.de 
neural activities can offer valuable insights for successful clinical applications of prosthetic vision. It is essential to inspect the spatial extent of retinal ganglion cell (RGC) responses to understand the limitations in spatial resolution that are experienced by implanted patients. Retinal stimulation can be roughly divided into two subgroups - epiretinal and subretinal - based on which side of the retina the electrodes are placed [6]. As is common, using a monopolar electrode configuration in which the return electrode is implanted in a distant part of the eye or body, the flow of current through the retina near the electrode is approximately perpendicular to the surface of the retina [7]. Accordingly, for examinations of the spatial spread of electrical stimulation, the two configurations can be considered equivalent to a first approximation [8].

The spatial extent (characterized by the electrical point spread function, EPSF) is important because it dictates the limits of electrical cross-talk for multiple electrodes and ultimately the electrode spacing of an implant. In recent decades, many research groups have investigated the spatial extent of RGCs evoked by single electrode or spatially patterned stimulation. Stett et al. recorded spike activity from the chick retina with a dense hexagonal microelectrode array (Hex-MEA), with varying electrode diameters $(10,20,30 \mu \mathrm{m})$ and interelectrode distances [9]. They were able to evoke responses up to $200 \mu \mathrm{m}$ away from the subretinal needle electrode and reported a half-width at half-maximal response (HWHM) of $100 \mu \mathrm{m}$ using an anodal voltage pulse. In an earlier study Stett et al used a bar-like spatial stimulation pattern $(0.8,1.2$ and $1.6 \mathrm{~V}$ amplitude) by selecting a row or a column on a $8 \times$ 8 MEA (diameter $10 \mu \mathrm{m}$, spacing $100 \mu \mathrm{m}$ ) which also yielded an increase in spike rate out to at least $100 \mu \mathrm{m}$ in the chick retina [10]. Eickenscheidt et al showed that rectangular cathodal epiretinal stripe-like stimulation (areas $100 \times 1000 \mu \mathrm{m}^{2}$, step spacing $50 \mu \mathrm{m}$ ) across a multicapacitor array can evoke responses in rabbit ON RGCs which extends over HWHM distance of $230 \mu \mathrm{m}$ [8]. Lorach et al. reported both the smaller $(\sim 100 \mu \mathrm{m})$ and larger $(\sim 250$ $\mu \mathrm{m})$ HWHM values in healthy and degenerated rat retina using a subretinal photovoltaic electrode array [11]. Similar distances for direct RGC stimulation were reported by Sekirnjak et al. who confirmed that RGCs as far as $160 \mu \mathrm{m}$ away can be stimulated in the rat retina [12]. Recently, our group used a MEA with $30 \mu \mathrm{m}$ electrode diameter and $200 \mu \mathrm{m}$ interelectrode distance to show that RGC responsiveness in wild type mice declines swiftly from $200 \mu \mathrm{m}$ to $283 \mu \mathrm{m}$ [13]. Importantly, however, we found reliable RGC responses in a small subset of RGCs even for distances up to $1000 \mu \mathrm{m}$. This result has been confirmed by Stutzki et al. who found electrical responsiveness to extend out to 500-1000 $\mu \mathrm{m}$ [14]. Due to the large $200 \mu \mathrm{m}$ interelectrode spacing of this array, we were unable to sample responses more densely or at shorter distances from the stimulating electrode. Therefore, we set out to further study the spatial extent of electrical stimulation in the retina with a denser MEA using the same stimuli as in our previous study.

\section{Materials and Methods}

\section{Animals}

Adult wild-type C57Bl/6J (Jackson Laboratory, Bar Harbor, ME, USA), postnatal day 30-45 mice were used. The mice were kept under a standard white cyclic lighting. They had free access to food and water. All possible efforts to minimize the number of animals used and their suffering were made. All animal experimental procedures were approved by state authorities (Regierungspräsidium, Tübingen) and conducted under the supervision of the Tübingen University facility for animal welfare (Einrichtung für Tierschutz, Tierärztlichen Dienst und Labortierkunde) in accordance with the NIH Guide for the Care and Use of Laboratory Animals.

\section{Retinal Preparation}

Inhalation of $\mathrm{CO}_{2}$ was used to anesthetize each mouse. After checking for withdrawal reflex by a pinch of the tissue between toes, the mouse was euthanized by cervical dislocation. The eyes were then 
Hosseinzadeh et al.: Spatial Extent of Epiretinal Stimulation

removed under normal room light and placed in carbogenated $\left(95 \% \mathrm{O}_{2}\right.$ and $5 \% \mathrm{CO}_{2}$ ) artificial cerebrospinal fluid (ACSF) solution containing (in mM): $125 \mathrm{NaCl}, 2.5 \mathrm{KCl}, 2 \mathrm{CaCl}_{2}, 1 \mathrm{MgCl}_{2}, 1.25 \mathrm{NaH}_{2} \mathrm{PO}_{4}, 26 \mathrm{NaHCO}_{3}$ and 20 Glucose; $\mathrm{pH}$ 7.4. During the dissection process (under red light to reduce photoreceptor bleaching) the cornea, ora serrata, lens and vitreous body were removed. Next the optic nerve was cut at the base of the retina and finally the retina was detached from the pigment epithelium. Next all traces of vitreous material on the inner surface of the retina were removed. Retinas were maintained in carbogenated ACSF until needed. All recordings were performed with a planar microelectrode array (MEA). Retinas were cut in half and a retinal half was oriented and flattened onto the MEA, ganglion cell side down, with two miniature forceps and was allowed to adapt for $>30 \mathrm{~min}$. Care was taken not to damage the retina or MEA during this process. In order to create better contact between the retina and the electrodes, a dialysis membrane (CelluSep, Membrane Filtration Products Inc., Seguin, Texas, USA) mounted on a custom Teflon ring was lowered onto the retina [15]. The MEA was then placed under the preamplifier and continuously superfused with carbogenated ACSF ( $\sim \mathrm{ml} / \mathrm{min}$, Peristaltic Perfusion System PPS2 perfusion pump; Multi Channel Systems, Reutlingen, Germany) maintained at $33^{\circ} \mathrm{C}$ using both a heating plate and a heated perfusion cannula (HE-Inv-8 \& PH01; Multi Channel Systems).

\section{MEA and Data Acquisition System}

A planar MEA with hexagonal layout (Hex-MEA) containing 59 circular TiN electrodes (60HexaMEA40/10, diameter: $10 \mu \mathrm{m}$, interelectrode and row spacing: 40 $\mu \mathrm{m}$; Multi Channel Systems, Reutlingen, Germany) as shown in Fig. $1 \mathrm{~b}$ with electrode tracks insulated by $\mathrm{Si}_{3} \mathrm{~N}_{4}$ on a glass substrate was used for recording from RGCs. The electrodes had impedances of approximately $250 \mathrm{k} \Omega$ at $1 \mathrm{kHz}$. The data acquisition system consisted of the MEA60 system (MCS, Reutlingen, Germany) including a 60 channel preamplifier with integrated filters and a blanking circuit (MEA 1060-Inv-BC). The blanking circuit was used to reduce recording noise by disconnecting amplifiers during stimulation pulses. The MEA_Select software was used to ground any defective electrodes and to choose the stimulating electrode. All data collection was done using the MC_Rack program. The computer was also fitted with MC_Card data acquisition hardware, an RS-232 serial port via which MEA_Select communicated with the amplifier, and an analog input card to record stimulus trigger signals. The raw data was sampled at a rate of $50 \mathrm{kHz} / \mathrm{channel}$. Using a filter with a bandwidth of $1 \mathrm{~Hz}-3 \mathrm{kHz}$ and a gain of 1100 . To confirm the contact of the retina on MEA, the retina was imaged by a microscope-mounted camera (DCC1645C, Thorlabs Inc, Newton, New Jersey, USA).

\section{Stimulation}

A stimulus generator (STG 2008, Multi Channel Systems, Reutlingen, Germany) was used to generate electrical stimulus pulses that were delivered from the ganglion cell side of the retina (epiretinally) via only one of the 59 electrodes. Stimulating electrodes were chosen based on proximity to electrodes with robust neural signals to ensure a maximum number of recorded neurons. In these epiretinal experiments, electrodes of the Hex-MEA were used to simultaneously stimulate and record from the retina. The stimulus consisted of monophasic cathodic rectangular voltage pulses of $1000 \mu$ s duration with the following amplitudes 100, $300,500,1000,1500,2000$, and $2500 \mathrm{mV}$ (Fig. 1c). Stimulus pulses were safely below the safe charge injection limit specified by the manufacturer for these electrodes (16 mA for $1 \mathrm{~ms}$; corresponding to 4000 $\mathrm{mV}$ at an assumed impedance of $250 \mathrm{kOhm}$ ) curves. Above these limits, faradic reactions occur that will lead to electrolysis of the electrode. The $100 \mathrm{mV}$ stimuli were subsequently found to not be reliably delivered by the stimulator and, consequently, are not included in the analysis presented here.

At the beginning and end of each experiment, spontaneous activity was recorded for $\sim 30 \mathrm{~s}$. We presented 5 voltage blocks, proceeding in an up/down sequence containing 14 pulses, repeated 5 times with an interval of $20 \mathrm{~s}$ after each block of 70 pulses to allow the recovery of RGC responsiveness before visual stimulation. The post-time setting in MC_Stim was set to $100 \mu$ s to facilitate active discharge of the stimulating electrode. Additionally, a wait time of $1 \mathrm{~ms}$ was set in the MEA_Select software to extend the blanking period before recording was resumed - thus minimizing stimulation artifacts.

To better sample various distances, upon completion of the full experiment, the electrical stimulation was often moved to a new electrode location and another full experiment was conducted with the same tissue. Accordingly, some cells yielded data from activation of multiple separate electrodes - each delivering a stimulus during a different, but sequential experiment. The reported distances are the distance from the 
Hosseinzadeh et al.: Spatial Extent of Epiretinal Stimulation

stimulating electrode and the electrode on which the cell was recorded. Of the total 58 cells recorded, 5 yielded samples from 5 separate stimulating electrodes and 28 yielded samples from 4 separate electrodes, the remaining 25 cells were only sampled once. Each of the 162 sample was independently tested for responsiveness, yielding 76 separate electrically responsive samples, and only included in Fig. 4 if the cell was found to be responsive for that particular stimulating electrode.

To monitor tissue health, six visual stimulus blocks were interleaved before, after, and between electrical stimulation blocks. Each visual stimulus block consisted of a full-field 'flash' stimulus of $2 \mathrm{~s}$ maximum brightness (ON, 40 klx) followed by $2 \mathrm{~s}$ minimum brightness (OFF, 20 lx), cycled 25 times without pause. Visual stimuli were presented with a commercial projector (Acer k10, US and Canada) through a custom optical pathway.

\section{Data Processing and Analysis}

The stored raw data were processed using commercial spike sorting software (Offline Sorter, Plexon Inc, TX, USA). To process the data, voltage traces were first high-pass filtered with a 12-pole Bessel filter with a cut off frequency of $51 \mathrm{~Hz}$. Following this, putative action potential events (spikes) whose filtered amplitude was greater than four standard deviations below the mean were detected. These events were sorted into clusters with an automated routine (T-distribution expectation maximization) to assign noise events as well as spikes from up to 3 cells recorded on each electrode to their own separate 'units'. Finally, as a quality control step, the multiple automated sorting solutions were manually inspected and the best one was modified as necessary in order to minimize Type I and Type II errors in attribution of events to different sources. Only units with a distinct waveform, stability during the whole measurement, an interspike interval lockout corresponding to a neuronal refractory period, and cross-correlograms indicative of good sorting were considered to contain the spike train from a single RGC and included in the analysis presented here. Time stamps assigned to the detection threshold crossing of these sorted spikes were collected with NeuroExplorer (PlexonInc, TX, USA) and exported to MATLAB (The Mathworks, Natick, MA, USA) for further analysis.

In all experiments, we processed and analysed all RGC responses recorded on electrodes other than the stimulating and reference electrodes. For each cell, the spiking response was integrated over the interval spanning 10 to 100 milliseconds after pulse onset, and averaged across the 25 repetitions of each unique stimulus. Likewise, a spontaneous rate, chosen to best characterize the baseline firing rate within the context of ongoing electrical stimulation, was calculated from the $1 \mathrm{~s}$ of recording time before each pulse and averaged across all samples. The response integration window of 10 to 100 was chosen to exclude spikes generated by either direct RGC stimulation which have a latency $<10 \mathrm{~ms}[12,16,17]$ or spontaneous spikes which make a significant contribution at latencies $>100 \mathrm{~ms}[18,19]$.

Our data was collected from 58 well-isolated RGCs from 3 retina halves - each from a different mouse. Of these, 36 cells were responsive to at least one of the 7 voltages. The vast majority were only responsive to -2000 and $-2500 \mathrm{mV}$. In light of previous experiments [13], we interpret this to reflect less current for a given voltage due to smaller electrodes and higher impedance relative to previous experiments. A cell was considered 'responsive' if the average response to one of the voltages was above threshold (3 standard deviations above the spontaneous rate) and with a minimum average response rate of $60 \%$ - at least 3 spikes for every 5 stimulus repetitions. While matching voltage stimuli are separated into upward and downward sequences for Figure 3 to examine hysteresis, the upward and downward sequences were combined into a single voltage response function for Figure 4 and the associated analysis. All analyses and statistical tests were performed in MATLAB.

\section{Results}

The present observations demonstrate the spatial extent (from 0 to $300 \mu \mathrm{m}$ ) of networkmediated electrical activation of retinal ganglion cells (RGCs) with a dense Hex-MEA in the epiretinal electrode configuration (Fig. 1).

Raw voltage traces were filtered so that spike times relative to electrical stimulus pulses could be extracted (Fig. 2). The spiking response was integrated from 10 to $100 \mathrm{~ms}$ following 
Fig. 1. Experimental design. a) Stimulation was delivered epiretinally by placing the ganglion cell side of the retina on the microelectrode array. b) The hexagonal array (Hex-MEA) used enabled dense sampling of the spatial spread of electrical stimulation. c) Prior to and after each experimental block, spontaneous activity (SP) was recorded for $\sim 30 \mathrm{~s}$. We presented 5 electrical stimulation blocks (ES), proceeding in the up/down sequence of 14 pulses shown repeated 5 times per block, and 6 visual stimulation blocks (VS) with a stimulus of $2 \mathrm{~s} \mathrm{ON} \mathrm{(} \sim 40 \mathrm{klx})$ and $2 \mathrm{~s} \mathrm{OFF} \mathrm{(} \sim 20$ $\mathrm{lx}$ ) that was cycled 25 times per block. An interval of about $20 \mathrm{~s}$ after each block was recorded in order to allow the recovery of RGC responsiveness. The electrical stimuli consisted of monophasic cathodic rectangular voltage pulses of $1000 \mu$ s duration with the following amplitudes, $-100,-300,-500,-1000,-1500$, -2000 , and $-2500 \mathrm{mV}$. The $100 \mathrm{mV}$ stimulus was not included in analysis because it could not be reliably delivered by the stimulator.

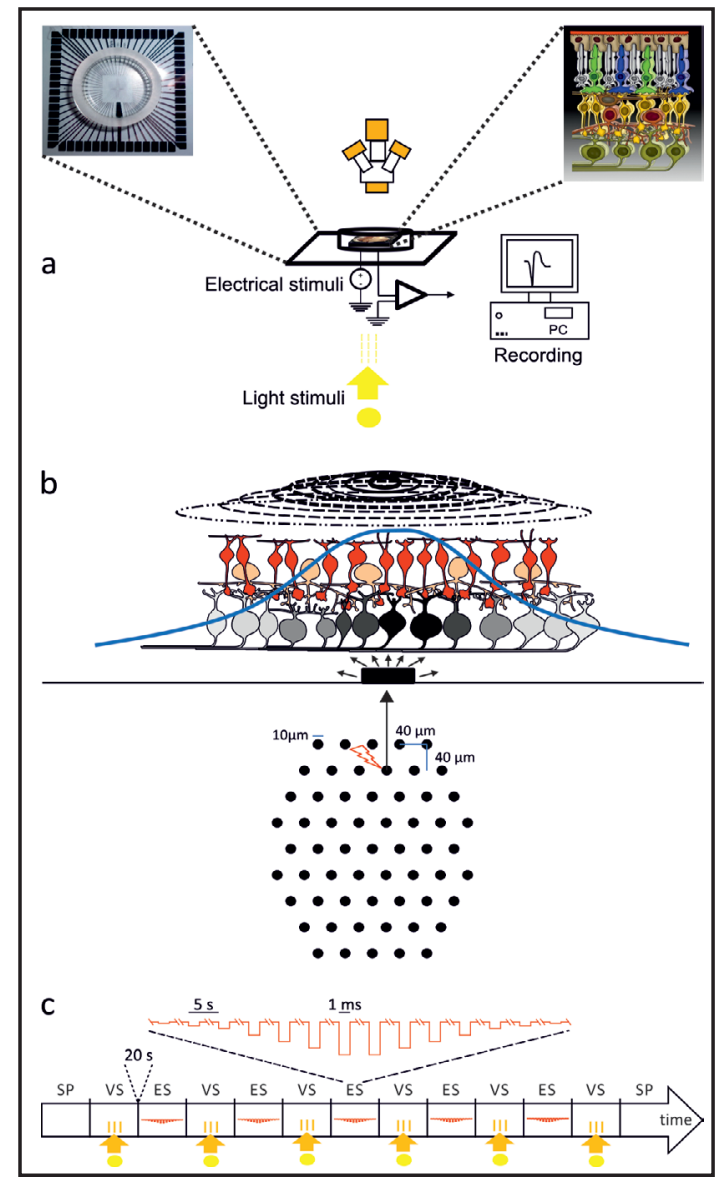

Fig. 2. Representative ganglion cell recording. a) Digitized voltage trace of responses to three stimuli. Stimulus artefacts indicated with arrows. Large positive deflections are perfusion drip artefacts. b) Zoom of the $300 \mathrm{~ms}$ surrounding the selected -2500 $\mathrm{mV}$ pulse from the upward half of the stimulus series. Spikes identified with arrows. c) Rastergram of all responses for this cell. The same spike train shown in b) are identified with arrows. d) Peristimulus time histogram binned at $2 \mathrm{~ms}$ intervals for all responses. Smoothed histogram (Gaussian smoothing filter, sigma $=4 \mathrm{~ms}$ ) is overlaid. Light grey bar covering b-d indicates the $10 \mathrm{~ms}$ excluded from response. Darker grey bar is the $90 \mathrm{~ms}$ of response integration. e) Voltage-response tuning curve during rising and falling phases of the stimulus sequence. Direction of sequence indicated by arrows. Error bars are standard deviation of the 25 repetitions.

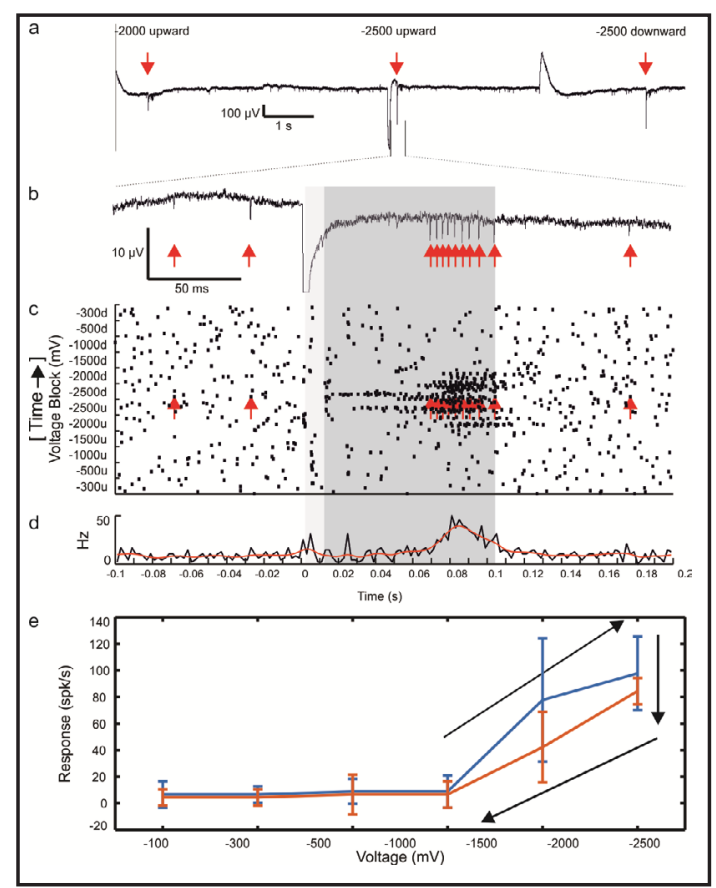

each pulse. This allowed us to examine the effects of indirect RGC stimulation via the activation of presynaptic neurons while excluding directly stimulated RGC spikes. Spikes with a latency 
Fig. 3. Voltage-response tuning curves showing response hysteresis. a) Normalized tuning curve of 76 individual RGC samples. After subtracting spontaneous firing rate for each cell, responses were normalized to the maximal response for that cell and averaged across all 25 repetitions. b). Mean +/- standard deviation across all normalized tuning curves. Supplemental time axis indicates elapsed time during the up/down sequence. Inset highlights hysteresis of $-2000 \mathrm{mV}$ responses from the upward and downward segments of the stimulus sequence. Lines are shaded according to whether the response increased or decreased. Thick line is the population mean.

longer than 100 ms were excluded because spontaneous spikes make a progressively larger contribution to the recorded response at such latencies. From Figure $2 b$ it is clear that while -2000 and $-2500 \mathrm{mV}$ stimuli elicited robust responses, weaker voltages did not. This result was consistent across our population, in contrast to previous reports [13]. After careful consideration, we judge the difference to result from the higher impedance of the electrodes used here as well as higher thresholds for such

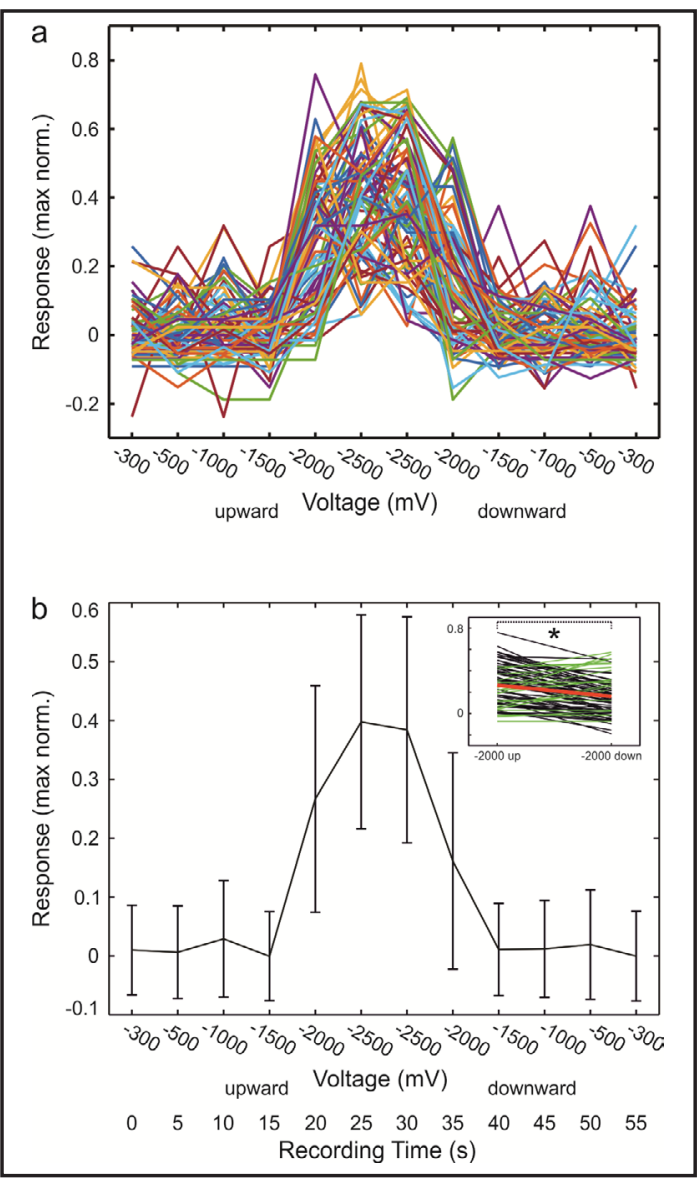
small electrodes. While the MEAs used in our previous experiments delivered $.57 \mathrm{mC} / \mathrm{cm}^{2}$ for a $1000 \mathrm{mV}$ pulse, the Hex-MEAs used here delivered $1.27 \mathrm{mC} / \mathrm{cm}^{2}$ for the same pulse. However, it has previously been shown that despite this 2-fold increase in charge density, the expected threshold increase for smaller electrodes would be 2-fold higher, if not more [12]. Electrical stimuli consisted of monophasic rectangular cathodic voltage pulses $(-300,-500$, $-1000,-1500,-2000$, and $-2500 \mathrm{mV}$ ) in an up/down sequence.

\section{Hysteresis}

The interpulse interval of 5 seconds was chosen to allow the retina to return to baseline activity between stimulus pulse intervals so that each response could be considered in isolation. Nevertheless, in the course of a previous study, we observed evidence that even such long intervals might not be sufficient to return the retina to baseline for electrical stimulation [13]. Therefore, in addition to investigating the spatial spread of electrical stimulation, we designed the present experiments in and up/down sequence to test whether electrical stimulus history at such long intervals influences responses. Hysteresis of the voltage response function demonstrating history dependence of the response can be seen for the example cell in Figure 2e. The 76 response samples of 36 cells (sometimes at multiple separate stimulating electrodes, see Methods) to the full up/down voltage sequence, averaged across 25 repetitions, are shown in Figure 3a. In the population average (Fig. 3b), it can more clearly be seen that responses to -2500 and $-2000 \mathrm{mV}$ were weaker when following stronger stimuli. The inset compares responses to $-2000 \mathrm{mV}$ during the rising and falling sequences. The average response decrease of $40 \%$ shown here was found to be statistically significant $(\mathrm{p}<0.05$; paired, one-tailed t-test) (Fig. 3b-Inset of the figure). Such differences in response to identical stimuli presented $15 \mathrm{~s}$ apart amounts to significant stimulus historydependent hysteresis in the voltage response tuning curve at a longer time interval than is 
Fig. 4. Spatial spread of electrical stimulation. a) Mean normalized firing rate of RGCs according to pulse amplitude and stimulation distance. b) Fraction of RGCs responsive to each pulse according to distance between stimulating and recording electrodes (see Methods for definition of 'responsive'). c) Mean population threshold vs. stimulation distance. Error bars in a) and b) are SD. Error bars in c) are 95\% binomial confidence intervals. Data were fit with linear ( $\mathrm{a}$ and b) and exponential (c) functions, shown along with dashed confidence bounds. Sample number is shown above the error bar for each distance.

currently considered to be relevant in retinal prostheses. Thus, RGCs have a 'memory' on the time scale of seconds for electrical stimulation. The implications of this long memory are examined in the Discussion.

\section{Spatial Extent}

We next examined how electrical responsiveness across the RGC population varies with distance from the stimulating electrode. Stronger voltages $(-2500$ vs. $-2000 \mathrm{mV}$ ) yielded stronger responses at a given distance as expected, with firing rates generally decreasing with increasing distance from stimulating electrode (Fig. 4a). We estimated the electrical point spread function (EPSF) by fitting a line to the data. From the fitted lines, we can estimate the halfwidth at half maximal response (HWHM) to be $303 \mu \mathrm{m}$ and $1034 \mu \mathrm{m}$ for -2000 and -2500 $\mathrm{mV}$, respectively. Using the Rayleigh criterion to calculate the limit of spatial resolution
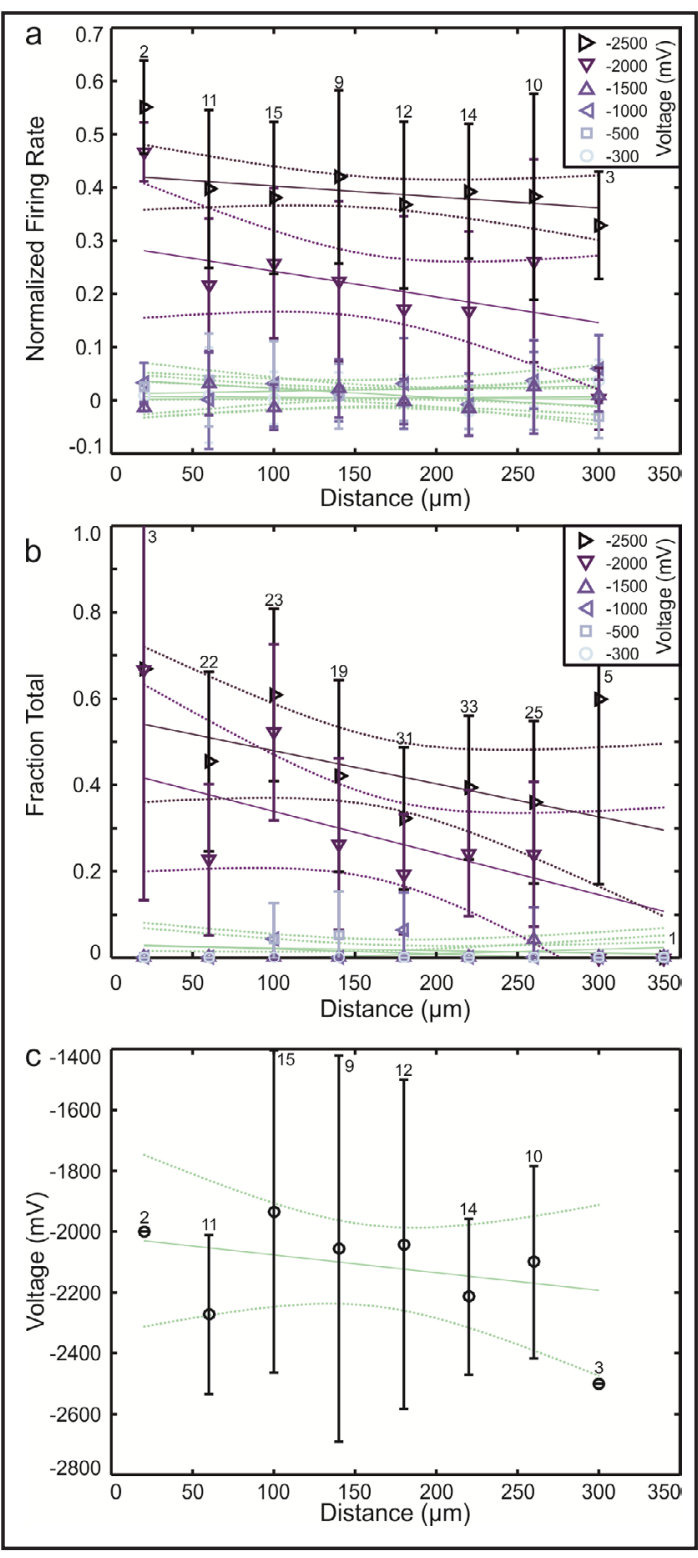
based on the HWHM of these EPSFs and assuming $288 \mu \mathrm{m} /$ degree in the human retina, we find corresponding acuities of 20/1263 or 1.80 logMAR for the $-2000 \mathrm{mV}$ curve and 20/4308 or $2.33 \log$ MAR for the $-2500 \mathrm{mV}$ curve [9].

To complement the analysis based on how firing rate varies with distance, we also examined how the fraction of electrically responsive RGCs varies with distance (Fig. 4b). As in Fig. 3, more RGCs were recruited with stronger voltages. Applying the same analysis methods we find HWHM to be 227 and $364 \mu \mathrm{m}$ for -2000 and $-2500 \mathrm{mV}$, respectively. Corresponding visual acuities are $20 / 946$ or $1.67 \log$ MAR for $-2000 \mathrm{mV}$ and $20 / 1517$ or $1.88 \log$ MAR for $-2500 \mathrm{mV}$. We can see from these and the previous results that spatial spread increases with stimulus amplitude, reducing the visual acuity that can be realized under otherwise ideal conditions. This supports the common practice of using the minimal level of stimulation necessary to yield a reliable visual percept.

Finally, we examined whether stimulation threshold varied with distance (Fig. 4c). We found a modest increase in threshold voltage magnitude with distance (about $-0.58 \mathrm{mV} / \mu \mathrm{m}$ from 0 to $300 \mu \mathrm{m}, \mathrm{r}^{2}=0.097$ ). We attribute this low correlation the fact that only 2000 and $2500 \mathrm{mV}$ pulses yielded responses from any RGCs with the present electrodes - resulting 
in the large error bars shown. To more precisely characterize how threshold changes with distance, we conclude that threshold amplitudes will need to be sampled much more finely in the future.

\section{Discussion}

The present observations describe the spatial spread of stimulation for RGCs of the wholemount retina in response to voltage pulses using high density electrode array. We show that voltage-response curves express hysteresis, reflecting adaptation to electrical stimulation. Evoked firing rate decreased irregularly from 0 to $300 \mu \mathrm{m}$, dependent on the strength of stimulation. The percentage of RGCs responsive to electrical stimulation also diminished from 0 to $300 \mu \mathrm{m}$ in a voltage dependent manner. Finally, we found little difference in threshold voltage across these distances.

Our results confirm earlier studies which all find a decrease in electrical response with increasing distance from the stimulating electrode $[8,9,20]$. According to our previous report, the fraction of responsive RGCs in wild type mice on a 'standard' MEA (30 $\mu$ m electrode diameter and $200 \mu \mathrm{m}$ interelectrode distance) diminished speedily from about $50 \%$ at 200 $\mu \mathrm{m}$ to $20 \%$ at $283 \mu \mathrm{m}$ with little additional drop up to $1000 \mu \mathrm{m}$ [13]. Nevertheless, we find inconsistencies in the literature regarding the choice of how to define a threshold response or the reporting of RGC recruitment versus the average RGC firing rate. Stimulation threshold, average response firing rate and the fraction of the RGC population recruited are all distinct but interconnected population measures of the extent of electrical stimulation. These are complimented by measurements of the electrical receptive fields of individual RGCs [8-10]. Because of the great diversity in RGCs and their responses to electrical stimulation, the choice of which measure to examine strongly impacts conclusions on the spatial limits of electrical stimulation. In order to better understand such matters we believe that better methods must be developed to differentiate the multitude of RGCs according to their 30 or more functional types [21] during in vitro electrical stimulation experiments. Only then can variability due to different cell types be controlled to uncover the true (presumably much lower) variability within distinct RGC types.

\section{Hysteresis}

The existence of hysteresis in electrical stimulation experiments has important implications for prosthetic stimulation research. The hysteresis we have reported implies a memory of at least 5 seconds (the interval between pulses). Ironically, this interval was chosen based on an awareness of such adaptational effects in an effort to yield fully independent responses. It has been shown that RGCs have a primary time constant of $150 \mathrm{~ms}$ [22]. Although their secondary time constant is $15 \mathrm{~s}$, it is so weak that we suspect adaptation within the retinal network to be the primary factor in the hysteresis observed here. In an absence of independence (hysteresis), researchers must take careful measures to account for adaptation to electrical stimuli. One method is to deliver 'probe' stimuli within the context of ongoing electrical stimulation [23]. An alternate method is to randomize the presentation of pulses, and average repetitions with different preceding stimulation histories [13]. Further research is needed to fully characterize the time course and strength of such adaptation effects for network-mediated RGC stimulation.

\section{Impedance Effects}

In striving to connect the present data with our previous experiments, we were reminded of the significant role that impedance plays in electrical stimulation - especially when the voltage rather than the current of each pulse is controlled. When voltage-controlled pulses are used, the actual current delivered is dependent on the impedance of the electrode/ tissue interface in accordance with Ohm's Law. This means that identical voltage pulses can 
deliver vastly different currents in arrays with high impedance such as old MEAs or MEAs with smaller electrodes (as in the present study). Likewise different retinal preparations or locations on the retina can have significantly different interface impedances. This variability amplifies the response variability already present in the diverse population of RGCs. In contrast, when current-controlled pulses are used, only the necessary voltage changes with impedance. This is significant because electrode current has a much more significant influence in driving neurons to response than electrode voltage. While the voltage gradient across neurons induces ion channels to open and produce an action potential, the voltage gradient across the retina is determined by the charge injected into the retina, not the voltage at the electrode surface which is strongly attenuated by a high-impedance electrode/tissue interface.

Nevertheless, even controlling for current density on the electrode, the threshold current and charge densities decrease significantly as impedance decreases (with increasing electrode diameter from 5 to $25 \mu \mathrm{m}$ ) [12]. This, combined with the higher impedance of smaller electrodes, explains why our previous experiments with $30 \mu \mathrm{m}$ electrodes (impedance $\sim 50 \mathrm{kOhm}$ ) yielded much lower and more varied thresholds than the present experiments with $10 \mu \mathrm{m}$ electrodes (impedance $\sim 250 \mathrm{kOhm}$ ). To control for such differences when stimulating with voltage-controlled pulses, we recommend matching the actual current delivered through different electrodes.

\section{Implications for Visual Acuity in Retinal Prostheses}

Stimulation of an implant's electrode activates a region of neurons. If this region is larger than the interelectrode spacing of the implant, crosstalk between electrodes occurs [24]. Even with significant crosstalk, phosphenes generated by neighbouring electrodes can be discriminated so long as their collective activation profile has sufficient contrast between electrodes to be detectable. The Rayleigh criterion which yields the resolution limit based on a point spread function is a useful boundary for two-point discrimination estimations.

Taken as a whole, the studies summarized in the introduction and the present results seem to indicate that the half-width at half-maximal response (HWHM) that characterizes the electrical point-spread function (ePSF) for stimulation of the population of retinal ganglion cells (RGCs) is approximately $250 \mu \mathrm{m}$. Within this population, a majority of RGCs seem to have smaller ePSFs with HWHM around $100 \mu \mathrm{m}$. Recently, Stutzki et al. have mapped the spatial extend of the electrical receptive field (eRF) for various RGCs [14]. The many single-cell receptive fields they report exhibit a broad range of ePSFs corresponding to those previously reported. Importantly, these eRFs also exhibit a wide range of spatial profile shapes as well as both onset and offset responses to electrical stimulation. Future work should continue to elaborate on such methods for probing the spatio-temporal response profile of individual RGCs. One such elaboration is the use of white-noise mapping which has the advantage that it is easily scalable to recording from tens to hundreds of simultaneously recorded RGCs. Although well-known in the world of visual neurophysiology, such linear systems methods have only recently been applied to visual prosthesis research $[23,25]$. As a better understanding of the dozens of different RGC types develops [21], it may become possible to target only RGCs with the smallest eRFs in order to provide the highest-possible prosthetic visual acuity.

Based on the current lower HWHM limit of $100 \mu \mathrm{m}$, it appears that the best visual acuity achievable stimulating RGCs with such ePSFs may be around 20/400 Snellen, or 1.3 logMAR. Indeed, the best visual acuity reported for a visual prosthesis is 20/546 or 1.43 logMAR [26]. However, it may be possible to achieve even better acuity. One possibility reflected in the present study is that the ePSF decreases modestly as stimulation amplitude decreases. By selectively activating a more homogeneous subset of the RGC population, it may be possible to fine-tune the stimulus such that it remains barely above threshold to produce the smallest possible ePSF. How small this may be first requires a full characterization of the electrical responses of the dozens of different RGC types. Just as importantly, given the 
many specializations of the human fovea where high-acuity vision is localized, it will soon be necessary to extend present research into primate retina.

At present, the highest resolution implants have electrodes spaced about $70 \mu \mathrm{m}$ apart $[11,26]$. If stimulation with such implants were to produce a field of mutually discriminable phosphenes, the best possible acuity would be 20/292 or $1.16 \log$ MAR. Such spacing is mostly limited by the need to separate relatively large electrodes on the order of $50 \mu \mathrm{m}$ in diameter. As electrodes using modern materials become smaller than this, stimulation threshold quickly approaches the limits of tissue and electrode damage. Therefore, although much work remains to minimize the ePSF, innovations in electrode materials and design will also be needed before perfect vision can be realized.

\section{Conclusion}

We have built upon previous studies by measuring the spatial extent of stimulation across the RGC population and applying an alternate method to characterize electrical responsiveness. In designing our experiments to also examine hysteresis of the voltageresponse function, we have demonstrated that network stimulation of RGCs is influence by stimuli at least 5 seconds prior. Accordingly, future research should carefully control for adaptation to electrical stimulation. We have affirmed that the pulse amplitude range used in retinal prostheses should be determined carefully by considering the potential reduction of spatial resolution which can be caused by undesirable stimulation of distant RGCs due to unnecessarily high stimulation amplitudes. In keeping with previous reports, we conclude that the spatial extent of electrical stimulation likely varies depending on the species, RGC type, and experimental methods used. Significantly more work will be needed to fully understand which spatial effects limit visual acuity in retinal prostheses and how they might be overcome.

\section{Funding}

Core funding for this study was provided by the German Federal Ministry of Education and Research (BMBF; 031 A 308). This study is part of the research program of the Bernstein Centre for Computational Neuroscience, Tuebingen, funded by the BMBF (01GQ1002). Additional support was received from the PRO RETINA foundation for prevention of blindness, the Tistou and Charlotte Kerstan Foundation, and the Werner Reichardt Centre for Integrative Neuroscience (CIN) at the Eberhard-Karls University of Tübingen. The CIN is an Excellence Cluster funded by the German Research Foundation (DFG) within the framework of the Excellence Initiative (EXC 307, including the senior professorship of Eberhart Zrenner).

\section{Acknowledgments}

The authors gratefully acknowledge the technical assistance of Kludija Masarini and Norman Rieger, and thank Regina Ebenhoch for retina graphics.

\section{Disclosure Statement}

None.

\section{References}

$>1$ Veraart C, Wanet-Defalque MC, Gerard B, Vanlierde A, Delbeke J: Pattern recognition with the optic nerve visual prosthesis. Artif Organs 2003;27:996-1004.

2 Dobelle WH: Artificial vision for the blind by connecting a television camera to the visual cortex. ASAIO J 2000;46:3-9. 
3 Schmidt EM, Bak MJ, Hambrecht FT, Kufta CV, O'Rourke DK, Vallabhanath P: Feasibility of a visual prosthesis for the blind based on intracortical microstimulation of the visual cortex. Brain 1996;119:507522.

4 Troyk P, Bak M, Berg J, Bradley D, Cogan S, Erickson R, Kufta C, McCreery D, Schmidt E, Towle V: A model for intracortical visual prosthesis research. Artif Organs 2003;27:1005-1015.

5 Merabet LB, Rizzo JF, Amedi A, Somers DC, Pascual-Leone A: What blindness can tell us about seeing again: merging neuroplasticity and neuroprostheses. Nat Rev Neurosci 2005;6:71-77.

- Zrenner E: Fighting blindness with microelectronics. Sci Transl Med 2013;5:210ps216.

-7 Gerhardt M, Groeger G, Maccarthy N: Monopolar vs. bipolar subretinal stimulation-an in vitro study. J Neurosci Methods 2011;199:26-34.

-8 Eickenscheidt M, Jenkner M, Thewes R, Fromherz P, Zeck G: Electrical stimulation of retinal neurons in epiretinal and subretinal configuration using a multicapacitor array. J Neurophysiol 2012;107:2742-2755.

-9 Stett A, Mai A, Herrmann T: Retinal charge sensitivity and spatial discrimination obtainable by subretinal implants: key lessons learned from isolated chicken retina. J Neural Eng 2007;4:S7-16.

10 Stett A, Barth W, Weiss S, Haemmerle H, Zrenner E: Electrical multisite stimulation of the isolated chicken retina. Vision Res 2000;40:1785-1795.

11 Lorach H, Goetz G, Smith R, Lei X, Mandel Y, Kamins T, Mathieson K, Huie P, Harris J, Sher A, Palanker D: Photovoltaic restoration of sight with high visual acuity. Nat Med 2015;21:476-482.

12 Sekirnjak C, Hottowy P, Sher A, Dabrowski W, Litke AM, Chichilnisky EJ: Electrical stimulation of mammalian retinal ganglion cells with multielectrode arrays. J Neurophysiol 2006;95:3311-3327.

13 Jalligampala A, Sekhar S, Zrenner E, Rathbun DL: Optimal voltage stimulation parameters for networkmediated responses in wild type and rd10 mouse retinal ganglion cells. J Neural Eng 2017;14:026004.

14 Stutzki H, Helmhold F, Eickenscheidt M, Zeck G: Subretinal electrical stimulation reveals intact network activity in the blind mouse retina. J Neurophysiol 2016;116:1684-1693.

15 Meister M, Pine J, Baylor DA: Multi-neuronal signals from the retina: acquisition and analysis. J Neurosci Methods 1994;51:95-106.

-16 Jensen RJ, Ziv OR, Rizzo JF: Responses of rabbit retinal ganglion cells to electrical stimulation with an epiretinal electrode. J Neural Eng 2005;2:S16-21.

17 Fried SI, Hsueh HA, Werblin FS: A method for generating precise temporal patterns of retinal spiking using prosthetic stimulation. J Neurophysiol 2006;95:970-978.

18 Boinagrov D, Pangratz-Fuehrer S, Goetz G, Palanker D: Selectivity of direct and network-mediated stimulation of the retinal ganglion cells with epi-, sub- and intraretinal electrodes. J Neural Eng 2014;11:026008.

19 Margalit E, Babai N, Luo J, Thoreson WB: Inner and outer retinal mechanisms engaged by epiretinal stimulation in normal and rd mice. Vis Neurosci 2011;28:145-154.

20 Ryu SB, Ye JH, Lee JS, Goo YS, Kim KH: Characterization of retinal ganglion cell activities evoked by temporally patterned electrical stimulation for the development of stimulus encoding strategies for retinal implants. Brain Res 2009;1275:33-42.

21 Baden T, Berens P, Franke K, Roman Roson M, Bethge M, Euler T: The functional diversity of retinal ganglion cells in the mouse. Nature 2016;529:345-350.

-22 Freeman DK, Fried SI: Multiple components of ganglion cell desensitization in response to prosthetic stimulation. J Neural Eng 2011;8:016008.

-23 Sekhar S, Jalligampala A, Zrenner E, Rathbun DL: Tickling the retina: integration of subthreshold electrical pulses can activate retinal neurons. J Neural Eng 2016;13:046004.

24 Zrenner E: Will retinal implants restore vision? Science 2002;295:1022-1025.

25 Freeman DK, Rizzo JF, Fried SI: Electric stimulation with sinusoids and white noise for neural prostheses. Front Neurosci 2010;4:28.

26 Stingl K, Bartz-Schmidt KU, Besch D, Braun A, Bruckmann A, Gekeler F, Greppmaier U, Hipp S, Hortdorfer G, Kernstock C, Koitschev A, Kusnyerik A, Sachs H, Schatz A, Stingl KT, Peters T, Wilhelm B, Zrenner E: Artificial vision with wirelessly powered subretinal electronic implant alpha-IMS. Proc Biol Sci 2013;280:20130077. 